\title{
Rodbertus' Angriff
}

\section{anf \\ Ricardos Renten-Theorie}

und der

\section{Lexis-Diehl'sche Rettungsversuch.}

\section{Inaugural-Dissertation}

zur Erlangung der Doktorwürde der hohen philosophischen Fakultät

der Königl. Christian-Albrechts-Universität zu Kiel

vorgelegt von

Dr. med. Franz Oppenheimer

aus Berlin.

Berlin 1908.

Druck von Georg Reimer. 
Zum Druck genehmigt:

Dr. Sudhaus,

z. Zt. Dekan.

1. Februar 1908. 